\title{
Modeling of optical devices: teaching modern optics to electrical engineers
}

\author{
Otto Schwelb
}

Otto Schwelb, "Modeling of optical devices: teaching modern optics to electrical engineers," Proc. SPIE 1603, Education in Optics, (1 March 1992); doi: $10.1117 / 12.57855$

SPIE. Event: Education in Optics, 1991, Leningrad, Russian Federation 
MODELLIKG OF OPTICAL DEVICES: TEACHIMG MODERM OPTICS TO FLECTRICAL BNGINIERS

\author{
Ot to Schwelb \\ Department of Electrical and Computer Engineering \\ Concordia University, Montreal, Quebec, Canada, H3G 1M8
}

\begin{abstract}
An outline is presented for a program to teach the modelling of optical components such as couplers, filters, resonators, interferometers, etc. to senfor electrical engineering students. The program exploits the student's background in linear algebra, circuit analysis and transmission line theory. It is designed to complement an elective course in microwave engineering. Numerous simulation examples associated with optical communication,
\end{abstract} measurement and sensing provide the experimental environment.

\title{
1. INTRODUCTION
}

The rapid progress of optical technology in such traditionally electrical and computer engineering (E.C.E.) disciplines as communications, digital signal processing, measurement and sensing, has made it imperative to include optical engineering in the E.C.E. curriculum. A workshop, held on July 25-27, 1990 near Estes Park, Colorado, recommended a number of topics, such as optoelectronics and laser electronics, to be part of the electrical engineering program.

Presently the E.C.E. student receives a good introduction into linear algebra, circuit theory and transmission line theory, and is given the opportunity through electives to familiarize herself with concepts in communications and microwave engineering. A great deal of optoelectronics material can be easily incorporated into the electives by making use of the students' background in the areas mentioned above.

The present paper describes a program and its technical details, implemented at the E.C.E. Department of Concordia University to teach undergraduates the operating principles of optoelectronic components, such as optical resonators, filters, interferometers and modulators of both bulk and surface wave architecture, using network models and concepts learned in microwave circuit design. In fact, optical and microwave engineering is taught within the same sequence of courses to emphasize their interdisciplinary nature and also to utilize study time more economically.

The program begins with fundamental concepts, such as representations of waves in terms of field components, representations of four-port circuits (scattering, scattering transfer, impedance transfer, etc.), and conservation principles (losslessness, reciprocity, bilateral symmetry, etc.). As we shall see later two-port and four-port circuits play a key role in the modelling of simple optical devices. It is therefore very important, that the student understands their properties. Next, elementary optical devices, used in communication, signal processing and industrial sensing are introduced. These include the lossless interface, the homogeneous delay line, or Fabry-Perot etalon, the beamsplitter, the directional coupler and the matched attenuator (neutral density filter). For each device both scattering and transfer 
representations are given. Students are assigned tasks to combine the basic components into composite devices, to investigate their characteristics and to check for violation of the conservation criteria. As a matter of course the student is introduced to the Michelson and Mach-Zehnder interferometers, the multimirror transmission line, or the stratified dielectric, the cascaded $\Delta \beta$ coupler, the unit transmittance interferometers and the frustrated total internal reflection (FTIR) filter.

With a reasonably good grasp of transmission line concepts the senior undergraduate can readily comprehend the spectral characteristics of periodic structures which constitute the next phase of the program. Here we refresh the student in the linear algebra of cascaded unit cells and solve problems related to grating structures, Bragg filters, reflective arrays and optical impedance matching. The principles of the transmission line equivalent of a unit cell (effective refractive index concept), and transverse resonance are developed in detail with illustrative examples drawn from the slab waveguide, the Bragg waveguide and the Fabry-Perot cavity.

It is shown that crystalline anisotropy is a cause of conversion between TE and TM modes and that as a result, a homogeneous anisotropic layer of finite thickness is analogous to a four-port coupler, while a semiinfinite one acts as a mode converting termination. In a graduate course these results are used to treat multilayer optical filters and birefringent antireflection coatings and to discuss guidance by superlattices.

Numerical design examples ąre assigned. Short simulation programs are written by students, using MATLAB ${ }^{2}$ or Mathcad ${ }^{3}$, in which they compute filter and impedance characteristics, $\omega-\beta$ diagrams, or performance characteristics as a function of material or geometrical parameters. The numerical assignments also draw attention to inaccuracies incurred in matrix manipulation and to the difficulties of finding the roots of complex transcendental equations. In the Laboratory students build and test some of the optical circuits encountered during the lectures. Video recordings of laboratory excercises provide additional support. The following sections outline the analytical framework of our program.

\section{FUNDAMENTAL CONCEPS}

The modelling process described in this paper applies to optical components, passive or active, which can be characterized by a two-port, or a four-port circuit, i.e. basically to two-dimensional problems. Included in this category are some uniform transmission lines, i.e. axially homogeneous lengths of isotropic or anisotropic media exhiblting gain or loss, the lossless interface or partially reflecting mirror, lumped element couplers such as the beamsplitter and the fused fiber coupler, distributed couplers, polarizers, Fabry-Perot resonators, and composite devices fabricated from the above. Excluded from this category are those components to which a valid $4 \times 4$ matrix description can not be applied because of the dominantly three-dimensional nature of the gegmetry. The description of the components is similar to the well known Jones and $4 \times 4$ mat:ix ${ }^{6}$ calculi, but they are modified to suit the background of electrical engineers. A variant of the $2 \times 2$ matrix analysis, using so-called $\triangle B C D$ matrices, is widely taught in laser electronics courses. Note also, that the analytical framework taught at Concordia applies to microwave and ultrasonics components as well. We exploit these analogies in a 
graduate course in Integrated Surface Wave Devices.

To utilize the scattering and wave transfer representations one must first define forward and backward running "waves" as appropriate linear combinations of electric and magnetic field components. Of the several definitions used in the literature ${ }^{8}$ we adopt the so-called travelling wave representation, normalized so that the power flow carried by a wave is given by its absolute value squared.

Consider a TE or $s$ polarized wave propagating in the $z$ direction in a homogeneous region characterized by a refractive index $n$. Assume, that the field components depend only on $x$ and $z$. Denoting the tangential field components of the total field (forward plus backward propagating) by $E_{y}$ and $H_{x}$, the incident and reflected waves $a^{+}(z)$ and $a^{-}(z)$ are defined by

$$
a(z)=\left[\begin{array}{c}
a^{+}(z) \\
a^{-}(z)
\end{array}\right]=\frac{1}{\sqrt{2}}\left[\begin{array}{cc}
\sqrt{Y}_{\mathrm{TE}} & \sqrt{Z}_{\mathrm{TE}} \\
\sqrt{Y} & -\sqrt{\mathrm{Z}} \\
\mathrm{TE}
\end{array}\right]\left[\begin{array}{c}
\eta_{0}^{-1 / 2} E_{\mathrm{y}} \\
-\eta_{0}^{1 / 2} H_{\mathrm{x}}
\end{array}\right] \exp \left(j k_{\mathrm{x}} \mathrm{x}\right)
$$

where $Y_{\mathrm{TE}}=n \cos (\theta)$ is the normalized wave admittance in the region, $\theta$ is the angle of incidence, $\eta_{0}=\sqrt{\mu_{0} / \varepsilon_{0}}$ is the free space impedance and the $\exp \left(j k_{x} x\right)$ factor is to cancel the conjugate factor present in $E_{y}$ and in $H_{x}$, in order to make $a^{+}$and $a^{-}$independent of $x$. A corresponding definition is used in the case of TM or $p$ polarized fields with a normallzed wave admittance of $Y_{T M}=n / \cos (\vartheta)$.

Having thus defined forward and backward running waves the scattering matrix of a two-port, illustrated in Fig.1, is defined by

$$
\mathbf{b}=\mathbf{S a}
$$

where $b=\left[\begin{array}{ll}b_{1} & b_{2}\end{array}\right]^{\mathrm{T}}$ and $a=\left[\begin{array}{ll}a_{1} & a_{2}\end{array}\right]^{\mathrm{T}}$ are the terminal parameter column vectors and

$$
S=\left[\begin{array}{ll}
S_{11} & S_{12} \\
S_{21} & S_{22}
\end{array}\right]
$$

This definition can be extended to any number of terminal pairs. In particular, the scattering matrix of an anisotropic layer supporting hybrid modes, or that of a device carrying both $T E$ and $T M$ modes is, like for a four-port coupler, a $4 \times 4$ matrix.

In addition to the "wave" basis we of ten use also the "voltage-current" basis, namely $\mathbf{g}(z)$, comprising the field components normal to the direction of propagation. The relationship between $g(z)$ and $a(z)$ is defined by

$$
\boldsymbol{g}(z)=\Omega \mathbf{a}(z)
$$

where 


$$
\Omega=\frac{1}{\sqrt{2}}\left[\begin{array}{cc}
\sqrt{Z} & \sqrt{Z} \\
\sqrt{Y} & -\sqrt{Y}
\end{array}\right]
$$

The voltage-current basis has the advantage of beeing continuous at dielectric interfaces, whereas the wave basis allows one to evaluate performance characteristics such as insertion loss and return loss. The port designations and the nomenclature for the waves, voltages and currents for a four-port device are given in Fig.2.

Devices consist usually of concatenated elements, it is therefore necessary to represent components by transfer matrices. Of these, we use most of ten the impedance transfer, or $\triangle B C D$ matrix, denoted $Q$ for brevity, and the scattering transfer matrix, denoted $A$. Referring to Fig. 2 and suppressing the omnipresent $\exp \left(j k_{x} x\right)$ factor, these representations are defined by

$$
g(0)=\operatorname{Qg}(1) \text { and } \mathbf{a}(0)=\operatorname{Aa}(1)
$$

where, for the left hand terminal vectors

and

$$
g(0)=\left[\eta_{0}^{-1 / 2} E_{y}(0) \eta_{0}^{-1 / 2} E_{x}(0)-\eta_{0}^{1 / 2} H(0) \eta_{0}^{1 / 2} H(0)\right]^{\mathrm{T}}
$$

$$
a(0)=\left[a_{1}^{+}(0) a_{1}^{-}(0) a_{2}^{+}(0) a_{2}^{-}(0)\right]^{T}=\left[\begin{array}{llll}
a_{1} & b_{1} & a_{2} & b_{2}
\end{array}\right]^{T}
$$

and corresponding definitions apply to the right hand terminal vectors at $z=1$. To give the student greater flexibility in analysis and design, and to prepare her for the diverse approaches encountered in the literature, we introduce some variants of the scattering transfer matrix $A$, namely $\boldsymbol{k}=\boldsymbol{A}^{-1}$ and $T$, the latter beeing defined by

$$
\left[\begin{array}{llll}
a_{1} & a_{2} & b_{1} & b_{2}
\end{array}\right]^{\mathrm{T}}=\mathrm{T}\left[b_{3} b_{4} a_{3} a_{4}\right]^{\mathrm{T}}
$$

Note, that $I=\Pi_{23} A_{23}$ where $\Pi_{23}$ is the permutation matrix obtained by switching columns 2 and 3 of the $4 \times 4$ identity matrix. As part of the introductory excercises, the student is given a conversion table for the $2 \times 2$ and $4 \times 4$ representations and invited to prepare a computer program to implement it.

To analyze distributed parameter systems incremental descriptions are used. On a wave basis, the set of first order differential equations, expressing the incremental variation of the coupled modes is

$$
\partial \mathbf{a}(z) / \partial z=-j \mathbf{R}_{\mathbf{a}} \mathbf{a}(z)
$$

whereas the corresponding set of linear differential equations, on a voltagecurrent basis is

$$
\partial \mathbf{g}(z) / \partial z=-j \mathbf{R}_{\mathbf{g}} \mathbf{g}(z)
$$

Here $R_{a}$ and $R_{g}$ are the appropriate $4 \times 4$ incremental, or coupling matrices which account for the coupling mechanism.

Optical devices usually satisfy certain conservation properties such as losslessness, reciprocity and bidirectional symmetry. These properties are reflected in the structure of their matrix representations. ${ }^{\text {Thus, for }}$ example, the scattering matrix of a reciprocal circuit is symmetric, that of a lossless circuit is unitary and the $R_{a}$ matrix of a bilaterally symetric 
four-port must satisfy

$$
\mathbf{R}_{a}=-\left[\begin{array}{llll}
0 & 1 & 0 & 0 \\
1 & 0 & 0 & 0 \\
0 & 0 & 0 & 1 \\
0 & 0 & 1 & 0
\end{array}\right] \mathbf{R}_{a}\left[\begin{array}{llll}
0 & 1 & 0 & 0 \\
1 & 0 & 0 & 0 \\
0 & 0 & 0 & 1 \\
0 & 0 & 1 & 0
\end{array}\right]
$$

These criteria are built into our simulation programs to provide an automatic checking facility for any undue violation of the conservation laws caused by programing or arithmetic errors.

Another fundamental concept is the principle of resonance. Resonance is the operating condition of lasers, Fabry-Perot cavities and, in the cross sectional plane, guiding structures. In the latter case we speak of transverse resonance. ${ }^{10}$ The principle of resonance can be conveniently expressed in terms of a transfer matrix. When, for example, a two-port circuit resonates as a result of reflective terminations as shown in Fig.1, then

$$
A_{11}+\Gamma_{\mathrm{R}} A_{12}-\Gamma_{\mathrm{L}} A_{21}-\Gamma_{\mathrm{L}} \Gamma_{\mathrm{R}} A_{22}=0
$$

must be satisfled, where $A_{1 j}$ are the elements of $A$ and $\Gamma_{L}$ and $\Gamma_{R}$ are the reflection coefficients of the left and right terminations, respectively. The resonance criterion in (10) can be readily generalized to four-port circuits, as illustrated in Fig.2. In this case, $\Gamma_{L}$ and $\Gamma_{R}$ are $2 \times 2$ matrices defined by

$$
\left[\begin{array}{l}
a_{1} \\
a_{2}
\end{array}\right]=\Gamma_{L}\left[\begin{array}{l}
b_{1} \\
b_{2}
\end{array}\right] \text { and }\left[\begin{array}{c}
a_{3} \\
a_{4}
\end{array}\right]=\Gamma_{R}\left[\begin{array}{l}
b_{3} \\
b_{4}
\end{array}\right]
$$

and the condition of resonance becomes

$$
\operatorname{det}\left[T_{A}+T_{B} \Gamma_{R}-\Gamma_{L} T_{C}-\Gamma_{L} T_{D} \Gamma_{R}\right]=0
$$

where the $4 \times 4 T$ matrix of the circuit has been partitioned into $2 \times 2$ submatrices according to

$$
T=\left[\begin{array}{cc}
T_{A} & T_{B} \\
T_{C} & T_{D}
\end{array}\right]
$$

The threshold of oscillation in a gain medium for example, is reached when the existence of outgoing waves does not depend on the presence of incoming waves. In this case $\Gamma_{L}=\Gamma_{R}=0$ and resonance sets in when ${ }^{11}$

$$
\operatorname{det}\left[T_{A}\right]=T_{11} T_{22}-T_{12} T_{21}=0
$$

In the following section we describe some of the applications presented to the student during the course.

\section{APPLICATIONS}

The first application introduced is the transfer matrix of the interface between two dielectric regions, denoted 1 and 2 . Since the boundary condition requires that $\mathbf{g}(1)=\mathbf{g}(2)$ and considering that $\mathbf{g}(i)=\Omega(i) \mathbf{a}(i), i=1,2$ therefore $a(1)=\Lambda a(2)$, where 


$$
A=\Omega^{-1}(1) \Omega(2)=\frac{1}{2}\left[\begin{array}{cc}
\sqrt{Y_{1} Z_{2}}+\sqrt{Z_{1} Y_{2}} & \sqrt{Y_{1} Z_{2}}-\sqrt{Z_{1} Y_{2}} \\
\sqrt{Y_{1} Z_{2}}-\sqrt{Z_{1} Y_{2}} & \sqrt{Y_{1} Z_{2}}+\sqrt{Z_{1} Y_{2}}
\end{array}\right]
$$

The determinant of $A$ is unity. Expression (16) applies regardless of the polarization of the incident plane wave. Using the conversion table the student calculates the scattering matrix of the interface and verifies whether it coincides with that obtained from first principles, namely

$$
S=\left[\begin{array}{rr}
\rho & \tau \\
\tau & -\rho
\end{array}\right]
$$

where, for TE modes $\tau=\sqrt{\frac{Y_{2}}{Y_{1}} t}, \rho=r_{1}$ and for TM modes $\tau=\frac{\cos \left(\theta_{2}\right)}{\cos \left(\theta_{1}\right)} \sqrt{\frac{Y_{2}}{Y_{1}}} t_{\|}, \rho=r_{\|}$. The Fresnel formulas provide $t$ and $r$ for both polarizations, power conservation, $\rho^{2}+\tau^{2}=1$, remains always valid.

As a second application we treat a length of possibly lossy uniform dielectric with partially reflecting interfaces. This is a generic model for a number of optical components, such as the thick mirror, the Fabry-Perot etalon, a length of fiber, or the gap region of a prism coupler or FTIR filter. The transfer matrix of this device is obtained simply by multiplying the transfer matrices of the left interface $\left(A_{1}\right)$, the delay line $\left(A_{D}\right)$ and the right interface $\left(A_{I}^{-1}\right)$ in that order. Thus, assuming bilaterally symmetric construction for simplicity, the transfer matrix of the mismatched delay line of complex phase delay $\varphi=(\alpha+j \beta) 1$ is

$$
\begin{aligned}
A & =A_{I} A_{D} A_{I}^{-1}=\frac{1}{\tau^{2}}\left[\begin{array}{ll}
1 & \rho \\
\rho & 1
\end{array}\right]\left[\begin{array}{ll}
e^{j \varphi} & 0 \\
0 & e^{-j \varphi}
\end{array}\right]\left[\begin{array}{rr}
1 & -\rho \\
-\rho & 1
\end{array}\right]= \\
& =\left[\begin{array}{cc}
\cos \varphi+j \frac{1+\rho^{2}}{1-\rho^{2}} \sin \varphi & -j \frac{2 \rho}{1-\rho^{2}} \sin \varphi \\
j \frac{2 \rho}{1-\rho^{2}} \sin \varphi & \cos \varphi-j \frac{1+\rho^{2}}{1-\rho^{2}} \sin \varphi
\end{array}\right]
\end{aligned}
$$

and the corresponding scattering matrix is

$$
\mathbf{S}=\frac{1}{\cos \varphi+j \frac{1+\rho^{2}}{1-\rho^{2}} \sin \varphi}\left[\begin{array}{cc}
j \frac{2 \rho}{1-\rho^{2}} \sin \varphi & 1 \\
1 & j \frac{2 \rho}{1-\rho^{2}} \sin \varphi
\end{array}\right]
$$

Next the student is introduced to the modelling of forward (codirectional) and backward (contradirectional) couplers. These can be lumped or distributed. A beamsplitter e.g., is a lumped element backward coupler, whereas two channel waveguides in close proximity constitute a distributed forward coupler. Numerous other examples from optics, microwaves and ultrasonics can be listed. For lumped element couplers, as shown schematically in Fig.3, the scattering matrix can be obtained by simple inspection, after explaining to the student 
using both a physical (referring to microwave couplers) and an analytic $\left(\mathbf{S} \times \mathbf{S}^{\top}=\mathbf{I}\right)$ argument, that the coupled and the uncoupled outputs must be in phase quadrature. Thus, assuming matched ports and infinite directivity, the scattering matrices of a forward and a backward coupler are, respectively,

$$
\mathbf{S}_{F}=\sqrt{1-\gamma}\left[\begin{array}{cccc}
0 & 0 & \sqrt{1-K} & j \sqrt{K} \\
0 & 0 & j \sqrt{K} & \sqrt{1-K} \\
\sqrt{1-K} & j \sqrt{K} & 0 & 0 \\
j \sqrt{K} & \sqrt{1-K} & 0 & 0
\end{array}\right] \text { and } \mathbf{S}_{\mathrm{B}}=\sqrt{1-\gamma}\left[\begin{array}{cccc}
0 & j \sqrt{K} & \sqrt{1-K} & 0 \\
j \sqrt{K} & 0 & 0 & \sqrt{1-K} \\
\sqrt{1-K} & 0 & 0 & j \sqrt{K} \\
0 & \sqrt{1-K} & j \sqrt{K} & 0
\end{array}\right]
$$

where $K$ is the coupling coefficient and $\gamma(0<\gamma<1)$ allows for loss.

Distributed couplers are approached via the incremental description given in (8) which accounts for the evolution of $a(z)$ between $z$ and $z+d z$. For a matched forward coupler with perfect directivity and a coupling coefficient $c$

$$
\mathbf{R}_{a}=\left[\begin{array}{cccc}
\beta_{1} & 0 & -c & 0 \\
0 & -\beta_{1} & 0 & c \\
-c & 0 & \beta_{2} & 0 \\
0 & c & 0 & -\beta_{2}
\end{array}\right]
$$

indicating, that the coupled guides are not necessarily identical ( $\left.\beta_{1} \neq \beta_{2}\right)$, thereby allowing to treat electrooptic couplers. The differential equation is solved by determining the four eigenvectors and elgenvalues of $R_{a}: u_{i}$ and $\kappa_{i}$, $1=1$ to 4 , respectively, and writing the solution for a coupler of length $l$ in the form

$$
\mathbf{a}(1)=M(1) \mathbf{a}(0)
$$

where $\mathbf{M}(1)=\mathbf{U} \Lambda(1) \mathbf{U}^{-1}, \mathbf{U}$ is the common modal matrix of $\mathbf{R}_{\mathbf{a}}$ and $\boldsymbol{M}$, whose columns are $u_{1}$, and $\Lambda(z)=\operatorname{diag}\left[\exp \left(-j \kappa_{1} z\right) \exp \left(-j \kappa_{2} z\right) \exp \left(-j \kappa_{3} z\right) \exp \left(-j \kappa_{4} z\right)\right]$. Evaluation of and subsequent conversion to the scattering representation ylelds

$$
S_{F}=\left[\begin{array}{cccc}
0 & 0 & s_{13} & s_{14} \\
0 & 0 & S_{14} & S_{24} \\
S_{13} & S_{14} & 0 & 0 \\
S_{14} & S_{24} & 0 & 0
\end{array}\right]
$$

where $\quad S_{13}=\exp \left(-j \beta_{0} 1\right)\left(\cos (\zeta 1)-j \frac{\Delta \beta}{2 \zeta} \sin (\zeta 1)\right), \quad S_{24}=\exp \left(-j \beta_{0} 1\right)\left(\cos (\zeta 1)+j \frac{\Delta \beta}{2 \zeta} \sin (\zeta 1)\right)$. and $S_{14}=j \exp \left(-j \beta_{0} 1\right) \frac{\kappa}{\zeta} \sin (\zeta 1)$, with $\beta_{0} \frac{\beta_{1}+\beta_{2}}{2}, \quad \Delta \beta_{1}-\beta_{1}-\beta_{2}$ and $\zeta=\sqrt{\left(\frac{\Delta \beta}{2}\right)^{2}+\kappa^{2}}$. A similar route is taken to obtain the transfer and scattering matrices of distributed backward couplers.

At this point we digress to note, that a homogeneous anisotropic medium of thickness 1 can also be modelled as a distributed four-port coupler, mixing TE 
and TM modes. The incremental matrix which describes electromagnetic wave propagation in the $x$ and $z$ directions through an anisotropic layer with interfaces in the $y-z$ plane is obtained from the Maxwell 14 equations. For a dielectric medium in the so-called longitudinal orientation ${ }^{14}$

$$
\mathbf{R}_{a}=\left[\begin{array}{cccc}
\beta_{\mathrm{TE}} & 0 & c & -c \\
0 & -\beta_{\mathrm{TE}} & -c & c \\
c & c & \beta_{\mathrm{TM}} & 0 \\
c & c & 0 & -\beta_{\mathrm{TM}}
\end{array}\right]
$$

indicating, that there is coupling between TE and TM modes in both forward and backward directions. It turns out, that solutions in closed form for $M(1)$ and $S$ can be obtained with relative ease for most uniaxial and biaxial media encountered in technology. This advanced subject with many of its significant consequences is brought into reach of students by the elegant circuit modelling method.

Having developed the modelling of couplers we turn our attention to composite devices. In particular to couplers with feedback, performing as ring resonators or interferometers. The circuit models of these either consist of a four-port with two ports connected by a possibly mismatched and lossy delay line, like that described by (18) and (19), or it consists of a coupler with arms terminated by partially transmitting mirrors. The former configuration is used in ring interferometers ${ }^{15}$ and fiber-optic lattice signal processors ${ }^{16}$ the latter in optical sensors ${ }^{17}$ and fiber amplifiers ${ }^{18}$.

By manipulating the linear equations involving the scattering transfer matrix of the coupler, we find that the $2 \times 2$ A matrix of the two-port that results when two of the coupler ports, say 2 and 4 , are connected by a delay line is

$$
A=\left[A_{A}+A_{B}\left(A_{2}^{-1}-A_{D}\right)^{-1} A_{C}\right]
$$

where $A_{2}$ is the $2 \times 2$ transfer matrix of the feedback circuit, and the $4 \times 4 A$ matrix of the coupler has been partitioned much like $T$ in (14). Expressions similar to (25) are obtained when the feedback path connects ports 2 and 3 , or 3 and 4 . The coupler can be forward or backward, lumped or distributed. The resultant transfer and scattering matrices allow the student to simulate the wavelength characteristics and to optimize the device with respect to fabrication parameters such as coupling coefficient, delay line length, etc.

With respect to resonator configurations using partially reflecting mirrors, the student begins with the generic model shown in Fig.4. The mirrors, with reflection coefficients $\rho_{1}$ as seen from the coupler, are distanced from the coupler by delays $\varphi_{i}=(\alpha+j \beta) 1_{i}, i=1$ to 4 . The transfer matrix of the entire resonator is

$$
A=A_{L} A_{M} A_{R}=\left[\begin{array}{ll}
A_{1} A_{A} A_{3} & A_{1} A_{B} A_{4} \\
A_{2} A_{C} A_{3} & A_{2} A_{D} A_{4}
\end{array}\right]
$$

where $A_{Y}$, the transfer matrix of the coupler has been partitioned as in (14), 
$A_{L}=\operatorname{diag}\left[A_{1} A_{2}\right], A_{R}=\operatorname{diag}\left[A_{3} A_{4}\right]$,

and

$$
A_{1,2}=\frac{1}{V\left(1-\rho_{1,2}^{2}\right)}\left[\begin{array}{cc}
\exp \left(j \varphi_{1,2}\right) & -\rho_{1,2} \exp \left(-j \varphi_{1,2}\right) \\
-\rho_{1,2} \exp \left(j \varphi_{1,2}\right) & \exp \left(-j \varphi_{1,2}\right)
\end{array}\right]
$$

$$
A_{3,4}=\frac{1}{\sqrt{ }\left(1-\rho_{3,4}^{2}\right)}\left[\begin{array}{cc}
\exp \left(j \varphi_{3,4}\right) & \rho_{3,4} \exp \left(j \varphi_{3,4}\right) \\
\rho_{3,4} \exp \left(-j \varphi_{3,4}\right) & \exp \left(-j \varphi_{3,4}\right)
\end{array}\right]
$$

The analysis of more sophisticated optical, microwave and ultrasonic devices, such as sensors consisting of cascaded Fox-Smith resonators, channel selection filters, ultrasonic surface wave resonators or Bragg reflectors fabrlcated with isotropic or anisotropic media, including ferromagnetics, are assigned to the student as a term paper. Below we describe some of their results.

In Fig. 5 the insertion loss in $d B$ is plotted vs. the phase delay for couplers with matched feedback. IL1 applies to a lumped forward coupler where ports 2 and 3 have been connected, or to a lumped backward coupler where the connection is between ports 3 and 4. IL2 applies to lumped couplers regardless of directionality with ports 2 and 4 connected as in a Sagnac interferometer. For both curves the coupling coefficient is $K=0.6$, the loss coefficient is $\gamma=0.1$ and the loss coefficient of the delay line is 0.95 .

Fig. 6 simulates a ring resonator using a lumped element backward coupler where ports 3 and 4 are linked by a partially reflecting transmission line, i.e., one including imperfect mirrors. The coupling coefficient is $K=0.84$ and the reflection coefflcient is $\rho=0.2$. The diagram shows the insertion loss (IL) and the return loss (RL) in $d B$, as a function of the normalized frequency $(\beta)$. Notice, that the insertion loss reaches very large values within very narrow ranges of wavelength. The insertion loss maxima can be shifted by adjusting the value of $\rho$. Results have also been obtained on the distributed $\Delta \beta$ coupler, on cascaded Mach-Zehnder interferometers and on resonant tunelling through a superlattice.

\section{CONCLUSIONS}

An outline for teaching modern optical devices to electrical engineering students has been presented. The methodology relies heavily on schematic circuit representation and $4 \times 4$ matrix algebra. The treatment is compact, elegant and produces useful results rapidly. Interested educators are invited to evaluate the efficiency of our method on published analyses.

\section{ACKNOWLEDGMENT}

The author acknowledges the support of the Natural Sclences and Engineering Research Council of Canada, and of the Fonds pour la Formation de Chercheurs et l'Aide à la Recherche, Québec. 


\section{REFERENCES}

1. "Report of the Workshop on Optoelectronics Education," Estes Park, CO, July 25-27, 1990. Optical Soc. Am., Washington, D.C. 20036.

2. MATLAB is a registered trademark of The MathWorks, Inc., Natick, MA 01760 .

3. Mathcad is a registered trademark of MathSoft, Inc., Cambridge, MA 02139.

4. Shaoul Ezekiel, Video Demonstrations in Lasers \& Optics, Massachusetts Inst. of Technology, Center for Advanced Engineering Study, Cambridge, MA 02139-4391.

5. J.W. Simmons and M.J. Guttmann, States, Waves and Photons: A Modern Introduction to Light, Addison-Wesley Publ. Co. Reading, MA 1970, chapter 3.

6. P. Yeh, "Electromagnetic propagation in birefringent layered media," J. Opt. Soc. Am. vol.69, no. 5, pp.742-756, 1979.

7. A.E. Siegman, Lasers, University Science Books, Mill Valley, CA 1986, chapter 15.

8. H.J. Carlin and A.B. Giordano, Network Theory, Prentice-Hall, Inc., Englewood Cliffs, NJ, 1964, chapter 4.

9. O. Schwelb and R. Antepyan, "Conservation laws for distributed four-ports," IEEE Trans. Microwave Theory and Tech., vol.MTT-33, no.2, pp. 157-160, 1985.

10. W.L. Weeks, Electromagnetic Theory for Engineering Applications, J. Wiley \& Sons, New York, NY, 1964, section 3.6.

11. G. Björk and 0 . Nilsson, "A new exact and efficient numerical matrix theory of complicated laser structures: properties of asymmetric phase-shifted DFB lasers," J. Lightwave Technol. vol.LT-5, no.1, pp.140-146, 1987.

12. P. Urquhart, "Transversely coupled fiber Fabry-Perot resonator: theory," Appl. Optics, vol.26, no.3, pp.456-463, 1987.

13. J.E. Adair, "Coupled-mode analysis of nonuniform coupled transmission lines," Dept. of Elect. Eng., Electron Physics Lab., Univ. of Michigan, Ann Arbor, MI, Tech. Rept. 106, March 1968.

14. 0 . Schwelb, "Stratified lossy anisotropic media: general characteristics," J. Opt. Soc. Am. A, vol.3, no. 2, pp.188-193, 1986.

15. H. van de Stadt, "Ring interferometers with unit transmittance," Appl. Optics, vol.24, no.15, pp.2290-2292, 1985.

16. L.F. Stokes, M. Chodorow and H.J. Shaw, "All-single-mode fiber resonator," Optics Letters, vol.7, no.6, pp. 288-290, 1982.

17. E. Udd, ed., Fiber Optic Sensors, J. Willey \& Sons, New York, NY, 1991.

18. F. Sanchez, "Matrix algebra for all-fiber optical resonators," J. Lightwave Technol., vol.9, no.7, pp.838-844, 1991.

19. P. Yeh, "Resonant tunelling of electromagnetic radiation in superlattice structures," J. Opt. Soc. Am. A, vol.2, no.4, pp568-571, 1985. 


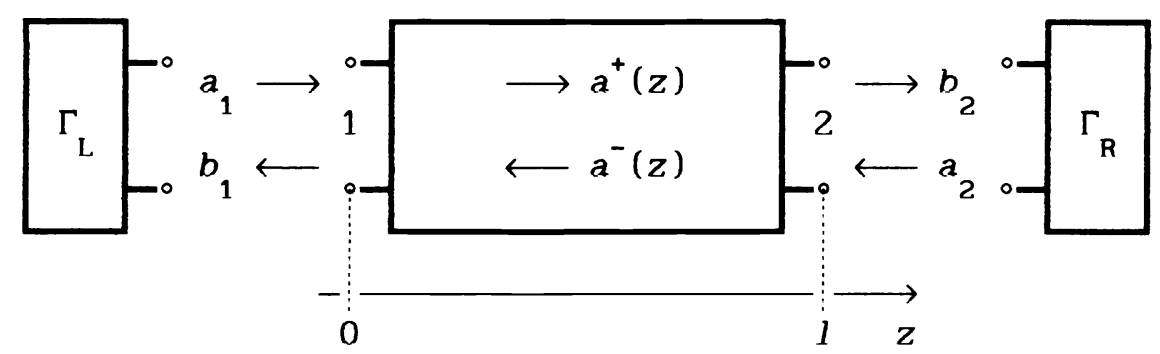

Fig.1. Schematic representation of a two-port device with terminal parameters and reflective terminations

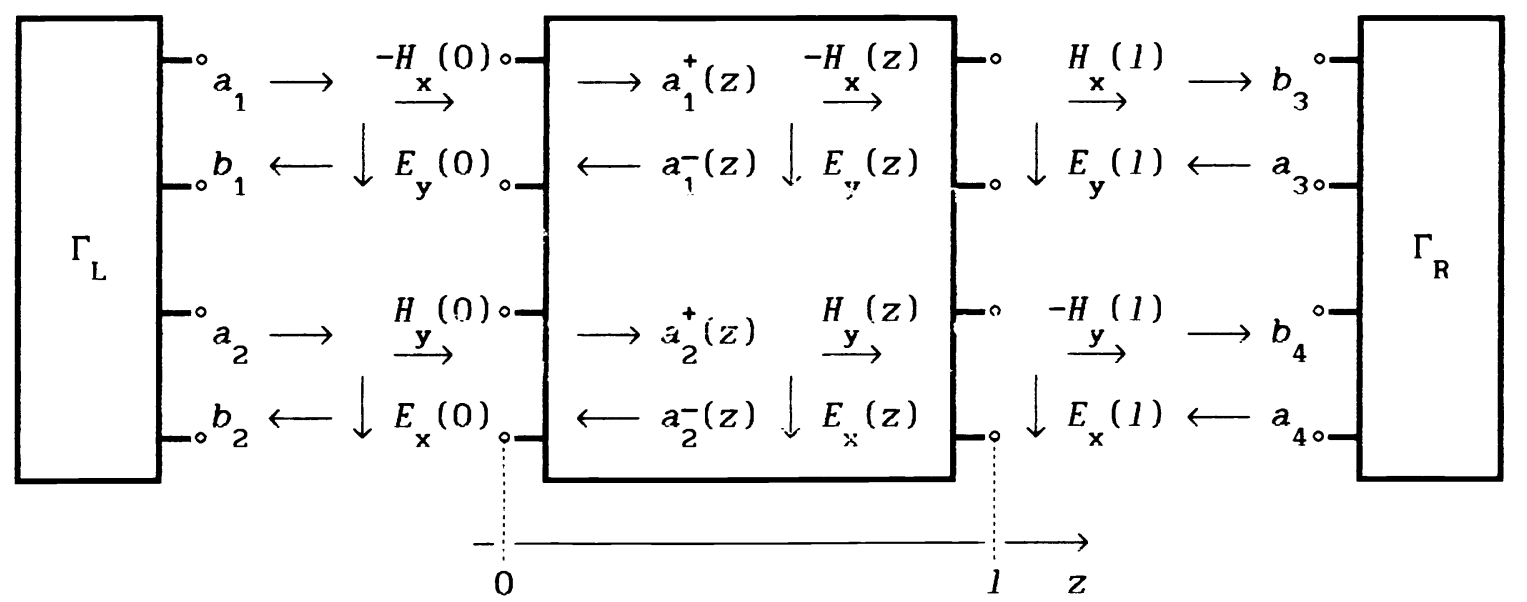

Fig.2. Schematic representation of a four-port device with terminal parameters and reflective terminations

a)

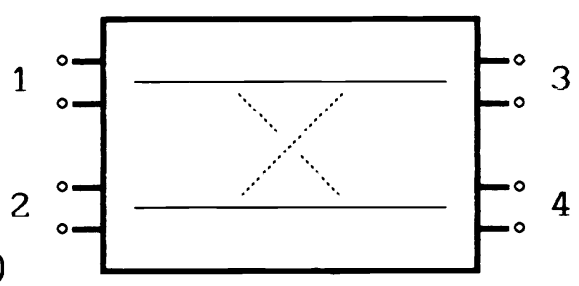

b)

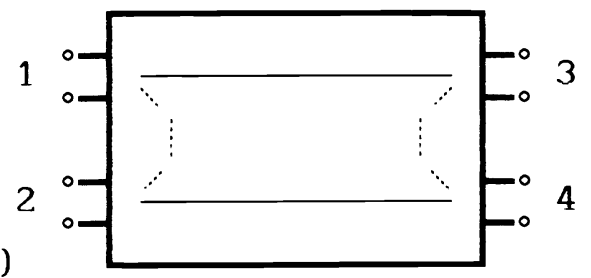

Fig. 3. Schematic diagrams of a) forward and b) backward directional couplers

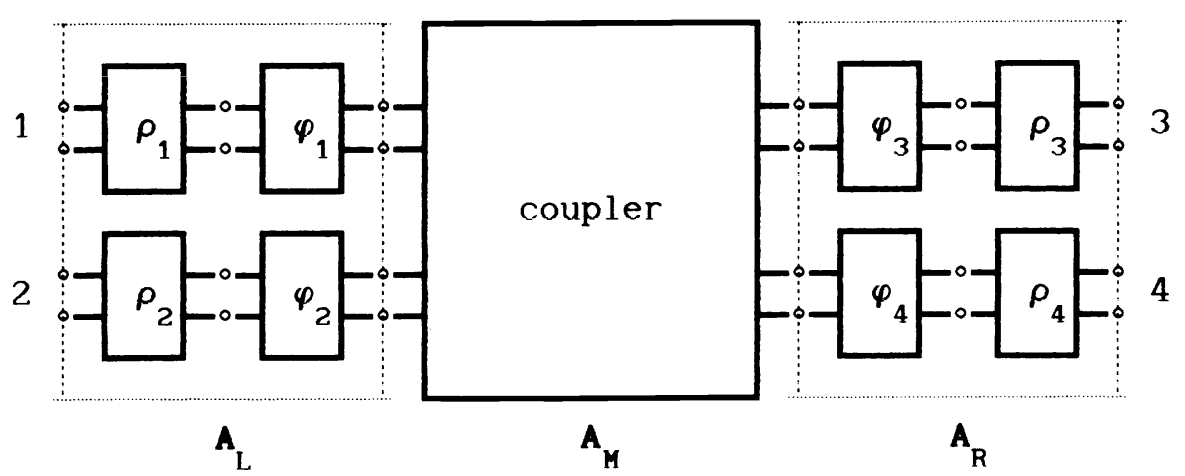

Fig.4. Model of a coupler terminated by partially reflecting mirrors (reflection coefficient $\rho_{i}$, as seen from the coupler), distanced from the coupler by delays $\varphi_{i}=(\alpha+i \beta) 1_{i}, i=1$ to 4 . 


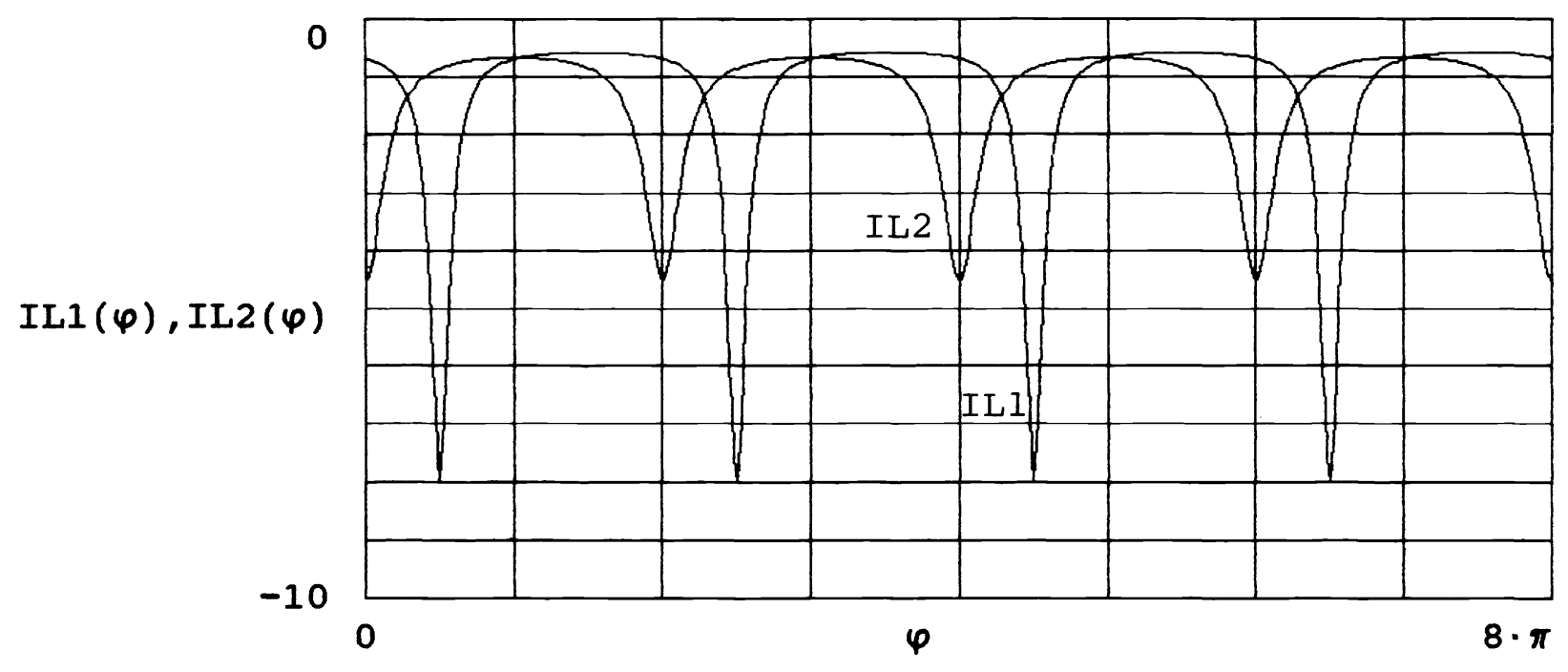

Fig.5. Insertion loss vs. phase delay for a coupler with two ports connected by a matched delay line. ILl: lumped forward coupler, ports 2 and 3 connected, IL2: lumped forward coupler, ports 2 and 4 connected. In both cases $K=0.6, \gamma=0.1$, transmission coefficient of delay line: 0.95 .

$I L(B), R L(B)$

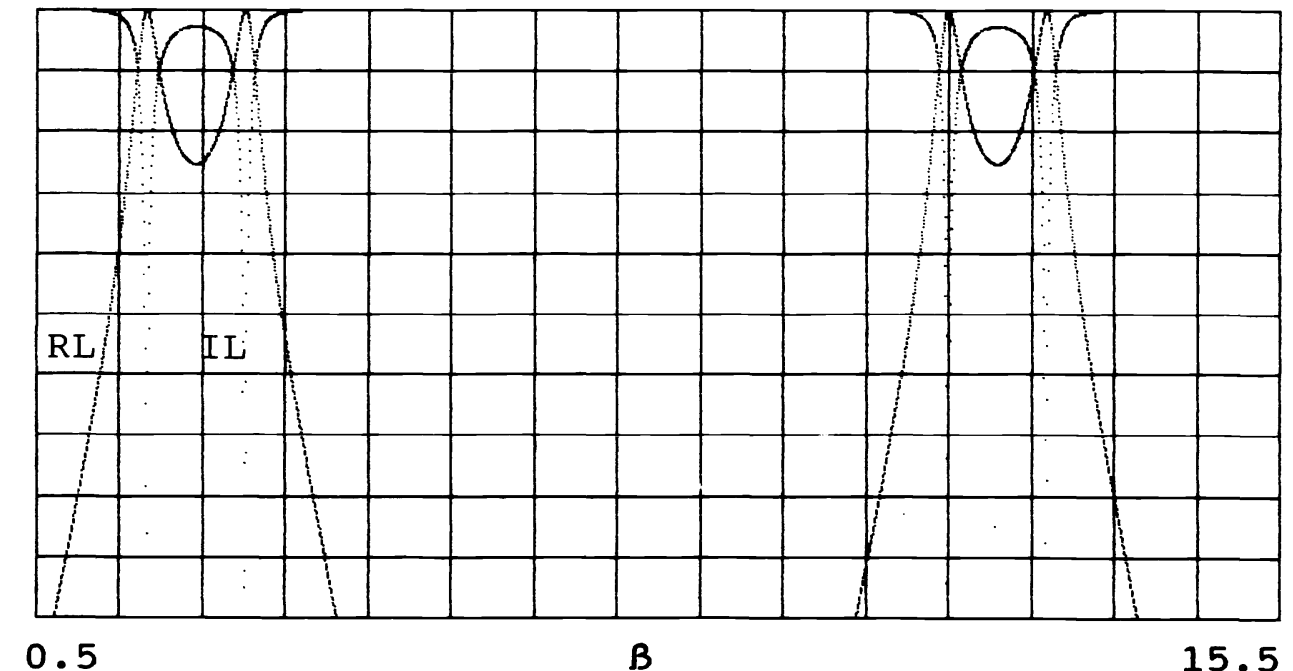

Fig.6 Insertion loss and return loss vs. B for a ring resonator consisting of a lumped element backward coupler with ports 3 and 4 connected by a mismatched delay line. $K=0.84, \rho=0.2$. 